Anaesthesist 2022 $\cdot 71: 426-436$ https://doi.org/10.1007/s00101-021-01064-w Eingegangen: 5. Mai 2021 Überarbeitet: 25. August 2021 Angenommen: 11. Oktober 2021 Online publiziert: 8. November 2021

(c) Der/die Autor(en) 2021

\section{Häufigkeitsverteilung und koordinative Umsetzung von Notfalloperationen in deutschen Krankenhäusern}

\section{Analyse in 26 Krankenhäusern in Abhängigkeit von der Versorgungsstufe}

\author{
Matthias Janda' $\cdot$ O. Karaca ${ }^{2} \cdot$ A. Brosin ${ }^{1} \cdot$ D. A. Reuter ${ }^{1} \cdot$ M. Schuster ${ }^{3}$ \\ ${ }^{1}$ Klinik und Poliklinik für Anästhesiologie und Intensivtherapie, Universitätsmedizin Rostock, Rostock, \\ Deutschland \\ ${ }^{2}$ digmed GmbH, Hamburg, Deutschland \\ ${ }^{3}$ Klinik für Anästhesiologie, Intensivmedizin, Notfallmedizin und Schmerztherapie, RKH-Kliniken Landkreis \\ Karlsruhe, Fürst-Stirum-Klinik Bruchsal und Rechbergklinik Bretten, Bruchsal, Deutschland
}

\section{Zusammenfassung}

Hintergrund: Die zeitgerechte Durchführung operativer Notfalleingriffe ist von enormer Bedeutung, da dies häufig direkten Einfluss auf die Morbidität und Letalität hat. Gemeinsame Empfehlungen der Fachverbände zur koordinativen Umsetzung liegen seit 2016 vor: N0 (Operation [Op.] sofort), N1 (Op.-Beginn im nächsten freien OP), N2 (Op.-Beginn $\leq 6 \mathrm{~h}$ ), N3 (Op. am Ende des Elektivprogramms), N4/dringlich (Op. innerhalb von 12-24h). Ziel dieser Studie war es, die Versorgungsrealität in deutschen Krankenhäusern unterschiedlicher Größe erstmals durch belastbare Daten differenziert zu beschreiben.

Methoden: Die Datenerhebung erfolgte in 26 Krankenhäusern unterschiedlicher Versorgungstypen über einen Zeitraum von 10 Tagen. Untersucht wurde die Häufigkeitsverteilung der einzelnen Notfallkategorien sowie die jeweilige Dauer von der Op.-Meldung bis zum Beginn der Anästhesie bzw. der Op. (Schnitt).

Ergebnisse: Insgesamt wurden 1603 Notfalleingriffe analysiert. Die durchschnittliche Dauer zwischen Op.-Meldung und Beginn der Anästhesie bzw. Schnitt betrug (min): N0 20,7 $\pm 14,3$ bzw. 43,6 $\pm 31,8$; N1 61,5 $\pm 48,7$ bzw. 90,1 $\pm 56,1$; N2 187,9 $\pm 152,0$ bzw. 220,5 $\pm 153,4$; N3 394,5 $\pm 392,3$ bzw. 428,3 $\pm 397,9$; N4/dringlich 494,8 $\pm 484,4$ bzw. 519,6 $\pm 486,6$. Die Verteilung der Notfallkategorien unterschied sich nicht signifikant zwischen Grund-, Regel- und Schwerpunktversorgern im Vergleich zu Maximalversorgern, einschließlich Universitätskliniken, $(p=0,731)$. Die Zeitdauern bis zur Realisierung des Notfalls unterschieden sich in Abhängigkeit vom Versorgungstyp nur gering und zeigten sich nur für N1 beim Beginn der Anästhesie sowie N3 beim Beginn der Anästhesie und beim Schnitt als statistisch signifikant.

Schlussfolgerung: Notfälle mit hoher Dringlichkeit werden in den teilnehmenden Häusern sehr zeitnah operativ versorgt. Die Unterschiede im Zeitmanagement zur Realisierung von Notfällen zwischen Grund-, Regel- und Schwerpunktversorgern im Vergleich zu Maximalversorgern, einschließlich Universitätskliniken, waren nur gering.

\section{Schlüsselwörter}

Krankenhausmanagement · Notfallmanagement · Notfallklassifikation · OP-Management · Prozesszeiten 
Aufstellung der teilnehmenden Krankenhäuser und lokalen Untersucher

Berlin: Charité - Campus Benjamin Franklin (M. Fischer, T. Schwithal), Charité - Campus Charité Mitte (J. Gerst, K. Bieber), Charité Campus Virchow-Klinikum (O. Birkelbach); Böblingen: Kliniken Böblingen ( $\mathrm{H}$. Lünig); Bretten: Rechbergklinik Bretten (O. Gültlinger); Bruchsal: Fürst-Stirum-Klinik Bruchsal (M.Schuster); Burgwedel: Klinikum Großburgwedel (K. Terhorst); Frankfurt: Krankenhaus Nordwest (S. Hieronymi); Göppingen: Klinik am Eichert Göppingen (C. Undeutsch); Göttingen: Universitätsmedizin Göttingen (M. Jipp); Hamburg: Universitätsklinikum Eppendorf (M. A. Punke); Hannover: Klinikum Siloah (L. Jünemann); Heilbronn: Klinikum am Gesundbrunnen Heilbronn (E. Hekler), Klinikum am Plattenwald Heilbronn (E. Hekler); Jena: Universitätsklinikum Jena (A. Gorse); Karlsruhe: Städtisches Klinikum Karlsruhe (W. Hummel); Korbach: Hessenklinik Stadtkrankenhaus Korbach (U. von Hagen); Ludwigsburg: Klinikum Ludwigsburg (H. Kursun, M. Schlam); Magdeburg: Universitätsklinikum Magdeburg (A. Mehlhose); Mainz: Katholisches Klinikum Mainz (M. Meyer); Neumünster: Friedrich-Ebert-Krankenhaus Neumünster (U. Goetzke); Offenbach: Sana Klinikum Offenbach (C. Smart); Ravensburg: Oberschwabenklinik St. Elisabeth Ravensburg (A. Scholz); Rostock: Universitätsmedizin Rostock (M. Janda, A. Brosin); Schweinfurt: Leopoldina-Krankenhaus Schweinfurt (A. Ertl); Siegen: St. Marien-Krankenhaus Siegen (D. Dills)

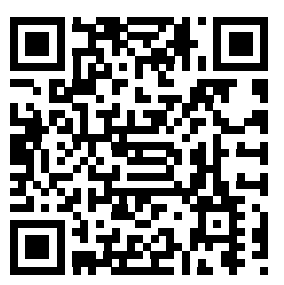

QR-Code scannen \& Beitrag online lesen
Insgesamt 7,1 der 18,8 Mio. in deutschen Krankenhäusern stationär behandelten Patienten wurden im Jahr 2019 operiert [10]. Auf Basis der European Surgical Outcomes Study (EuSOS) muss davon ausgegangen werden, dass sich rund 1,7 Mio. dieser Patienten aufgrund einer dringlichen oder vitalen Indikation einem ungeplanten operativen Eingriff unterziehen mussten [16]. Dabei stellt der Zeitpunkt der Operation einen wesentlichen Prädiktor für das Outcome des Patienten dar [15].

\section{Hintergrund}

Mit dem "Glossar perioperativer Prozesszeiten und Kennzahlen“ wurde 2016 erstmals eine gemeinsame Empfehlung des Berufsverbands Deutscher Anästhesisten (BDA), der Deutschen Gesellschaft für Anästhesiologie und Intensivmedizin (DGAI), des Berufsverbands der Deutschen Chirurgen (BDC), der Deutschen Gesellschaft für Chirurgie (DGCH) sowie des Verbandes für OP-Management (VOPM) zur Klassifikation von Notfalloperationen (Notfall-Op.) vorgelegt [3]. Dieses Positionspapier, aktuell veröffentlicht in der Version 2020 [1], enthält neben der einheitlichen Definition der medizinischen Dringlichkeit operativer Notfälle nachfolgende Handlungsempfehlung zur koordinativen Reaktion im operativen Tagesbetrieb unter Nennung einer definierten zeitlichen Dimension:

- N0: Eingriff höchster Dringlichkeit:

- Op. sofort, ggf. unmittelbar am Aufenthaltsort des Patienten (z. B. Schockraum, Intensivstation, Kreißsaal).

- N1: Sehr hohe Dringlichkeit:

- Op. auf dem nächsten freien geeigneten Tisch, unabhängig von der Fachdisziplin.

- N2: Op.-Beginn $\leq 6 \mathrm{~h}$ nach Meldung:

- Op. auf dem nächsten freien Tisch der eigenen Fachdisziplin. Soweit möglich, Abwarten der Nüchternheit.

- N3: Op. am Ende des Elektivprogramms im Rahmen des Notfallmanagements.

- N4/dringlich: Op. innerhalb 12-24h:

- Einpflegen in das Op.-Programm des Folgetags.
Diese konsentierte Empfehlung ermöglicht damit ein einheitliches, standardisiertes Vorgehen aller Beteiligten und somit erstmals eine Vergleichbarkeit des Managements von Notfall-Op. in Krankenhäusern unterschiedlichster Versorgungsstufen.

Ziel der vorliegenden Studie war es, die aktuelle organisatorische Versorgungsqualität bei der Umsetzung operativer Notfalleingriffe in Deutschland auf Grundlage objektiver Daten differenziert abzubilden. Vor diesem Hintergrund sollte insbesondere die Frage beantwortet werden, ob bei der zeitlichen Umsetzung in Abhängigkeit von der Größe des Krankenhauses sowie der behandelnden Fachabteilung Unterschiede existieren.

\section{Methodik}

In dieser prospektiven Studie hatten Krankenhäuser, welche sich am BenchmarkingProgramm des BDA, des BDC und des VOPM beteiligen, die Möglichkeit zur freiwilligen Teilnahme. Das BenchmarkingProgramm ermöglicht Krankenhäusern in Deutschland, Österreich und der Schweiz die strukturierte Auswertung und das Benchmarking von Op.-Prozess-Daten in einem Online-Tool [6]. Dieses Benchmarking-Programm besteht seit 2009, seither haben über 320 Krankenhäuser ihre Op.Prozess-Daten über das Online-Tool ausgewertet. Technisch realisiert wird das Benchmarking-Programm durch die digmed (Hamburg, Deutschland) $\mathrm{GmbH}$, die auch an der Datenerhebung und -analyse der hier vorliegenden Studie beteiligt war.

\section{Datenerhebung}

Die Erhebung der Daten erfolgte an 10 Werktagen im Zeitraum vom 27.01.2020 bis zum 07.02.2020, Wochenenden waren von der Erhebung ausgenommen. Die tägliche Erfassung der Parameter während des Erhebungszeitraums (innerhalb und außerhalb der Kernbetriebszeit) erfolgte mittels eines standardisierten Bogens, welcher webbasiert übermittelt wurde.

Erfasst wurden die Notfallmeldungen durch Dokumentation der meldenden operativen Fachabteilung sowie der Notfallklassifikation gemäß der gemeinsamen Empfehlung der Verbände [3]. 


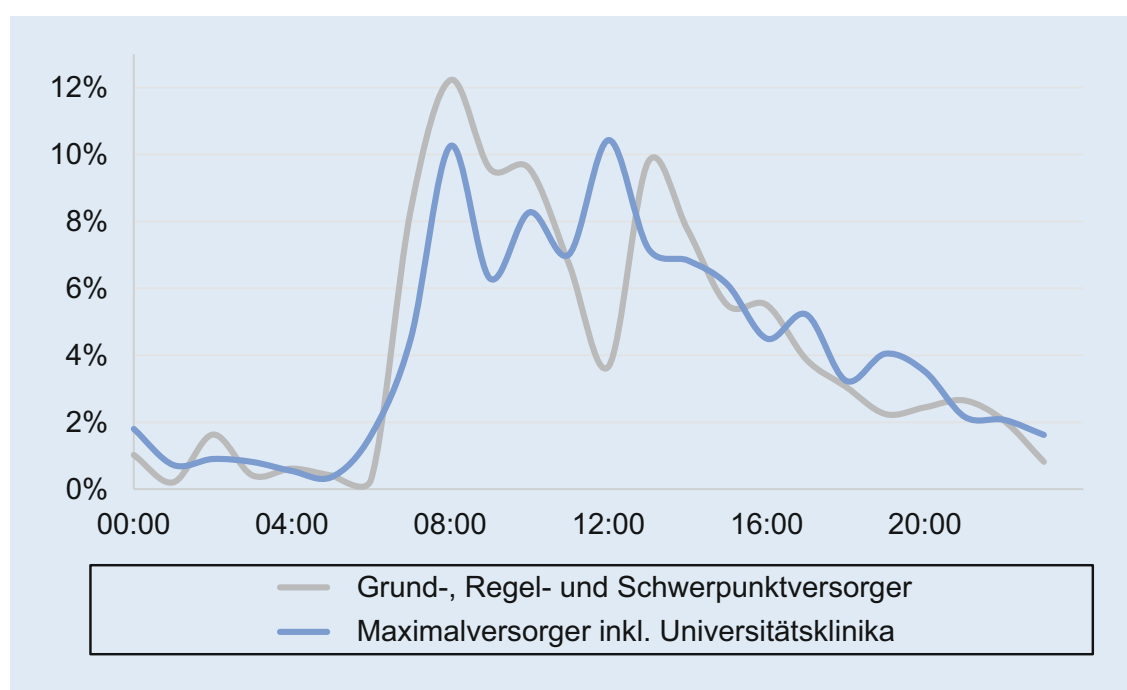

Abb. 1 \ Verteilung von operativen Notfalleingriffen im Tagesverlauf nach Versorgungsauftrag. Dargestellt ist die prozentuale Verteilung auf Basis des Zeitpunkts Op.-Anmeldung

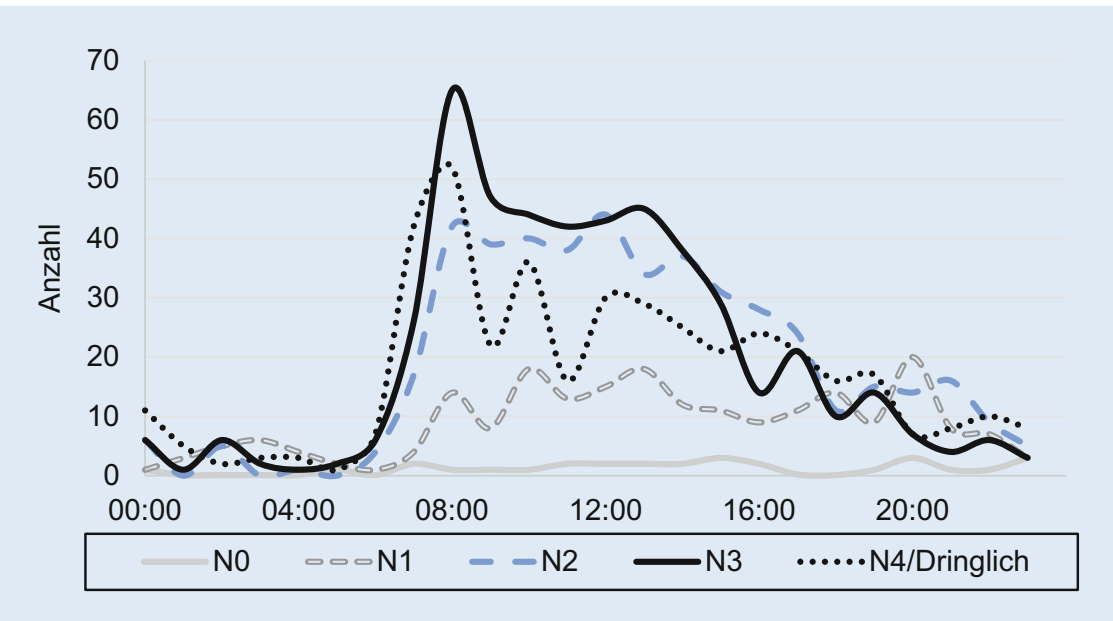

Abb. $2 \Delta$ Verteilung von operativen Notfalleingriffen im Tagesverlauf nach Notfallklassifikation. Dargestellt ist die Verteilung auf Basis des Zeitpunkts Op.-Anmeldung (absolute Anzahl der Fälle je Zeiteinheit)

Zur Darstellung der Umsetzung der Notfallmeldungen wurden nachfolgende Daten erhoben:

- Zeitpunkt der Anmeldung des Notfalleingriffs (telefonisch und/oder im Krankenhausinformationssystem respektive Op.-Dokumentation-System),

- Erfassung evtl. Änderungen der Notfallklassifikation (Hochstufung, Herabstufung, Abmeldung) vor Beginn der Umsetzung des Notfalleingriffs,

- Erfassung der Zeitpunkte „Beginn der Anästhesie" (definiert als Zeitpunkt der Injektion des ersten Narkosemedikaments bzw. bei Regionalanästhesie der Zeitpunkt der Hautpunktion, A7 laut

\section{Datenausschluss}

Um eine Konsistenz der Daten sicherzustellen und Fälle auszuschließen, welche einen vom normalen Notfall stark abweichenden Prozesspfad aufweisen, wurden Fälle mit den folgenden Kriterien von der weiteren Analyse ausgeschlossen:

- Der Schnitt lag vor dem Beginn der Anästhesie.

- Beginn der Anästhesie oder Schnitt lagen vor Anmeldung des Op.-Falls.

- Eine Differenz zwischen Beginn der Anästhesie und Schnitt von mehr als 120 min.

- Eine Differenz von der Anmeldung des Notfalleingriffs bis zum Beginn der Anästhesie $>48 \mathrm{~h}$ (N4/dringlich, N3), $>24 \mathrm{~h}(\mathrm{~N} 2)$, $>6 \mathrm{~h}$ (N1) bzw. $>90 \mathrm{~min}$ (NO).

Bei den ersten beiden Punkten handelt es sich um Notfälle, in denen das Behandlungsteam erst nach Beginn der Versorgung eines Notfalles dazu gerufen wurde. Bei den letzten beiden Punkten handelt es sich um Notfälle, in denen die Versorgung der Patienten einen anderen Notfallpfad genommen hat als ursprünglich gedacht, z. B. eine hochdringliche Notfallanmeldung, die sich im Weiteren als weniger dringlich erweist und zeitlich zu einem viel späteren Zeitpunkt versorgt wurde.

\section{Datenauswertung}

Für die Auswertung wurden die Krankenhäuser in 2 Gruppen unterteilt, mit dem Ziel, die Umsetzungspraxis an Häusern der Grund-, Regel- und Schwerpunktversorgung (Gruppe 1) mit der an Krankenhäusern der Maximalversorgung, einschließlich Universitätskliniken, (Gruppe 2) zu vergleichen.

Zunächst wurde für jedes Haus die prozentuale Zuordnung der Notfalleingriffe zu den Notfallklassifikationen vorgenommen. Davon ausgehend wurde untersucht, ob sich diese Verteilung in den vorgenannten Gruppen statistisch signifikant unterscheidet.

In einem weiteren Schritt wurde die Dauer der Umsetzung des operativen Notfalleingriffs analysiert. Hierzu wurden für jede Notfallkategorie folgende Zeitintervalle im Vergleich zwischen Häusern 
Tab. 1 Strukturmerkmale der teilnehmenden Krankenhäuser

\begin{tabular}{|c|c|c|}
\hline- & $\begin{array}{l}\text { Grund-, Re- } \\
\text { gel- und } \\
\text { Schwerpunkt- } \\
\text { versorger } \\
(n=12)\end{array}$ & $\begin{array}{l}\text { Maximalver- } \\
\text { sorger, inkl. } \\
\text { Universitäts- } \\
\text { klinika } \\
(n=14)\end{array}$ \\
\hline $\begin{array}{l}\text { Anzahl der } \\
\text { OPa }\end{array}$ & $8,4 \pm 4,5$ & $22,2 \pm 9,4$ \\
\hline $\begin{array}{l}\text { OP-Öff- } \\
\text { nungszeit } \\
\geq 10 \mathrm{~h}^{\mathrm{b}}\end{array}$ & $4(33,3 \%)$ & $9(64,3 \%)$ \\
\hline $\begin{array}{l}\text { Notfallkon- } \\
\text { zept vor- } \\
\text { handen }^{\text {b }}\end{array}$ & $4(33,3 \%)$ & $6(42,9 \%)$ \\
\hline \multicolumn{3}{|c|}{$\begin{array}{l}\text { 'Dargestellt sind die Mittelwerte ( } \pm \text { Standard- } \\
\text { abweichung) } \\
\text { 'Dargestellt sind die Anzahl der Häuser sowie } \\
\text { der prozentuale Anteil }\end{array}$} \\
\hline
\end{tabular}

der Grund-, Regel- und Schwerpunktversorgung und Häusern der Maximalversorgung, einschließlich Universitätskliniken, analysiert:

- Anmeldung der Notfall-Op. bis zum Beginn der Anästhesie (A7 laut Glossar),

- Anmeldung der Notfall-Op. bis zum Beginn der Op. $(08=$ Schnitt laut Glossar).

Ergänzend wurden diese Untersuchungen in 4 operativen Fachabteilungen separat durchgeführt, die typischerweise ein hohes Notfallaufkommen aufweisen und in den meisten Kliniken unabhängig von ihrer Größe vertreten sind [9]:

- Allgemeinchirurgie (ACH),

- Unfallchirurgie (UCH),
Tab. 2 Verteilung operativer Notfalleingriffe nach Notfallkategorie ${ }^{a}$

\begin{tabular}{|l|l|l|}
\hline- & $\begin{array}{l}\text { Grund-, Regel- } \\
\text { und Schwer- } \\
\text { punktversorger } \\
n(\%)\end{array}$ & $\begin{array}{l}\text { Maximalver- } \\
\text { sorger, inkl. } \\
\text { Universitätskli- } \\
\text { nika } \\
n(\%)\end{array}$ \\
\hline N0 & $8(1,6)$ & $21(1,9)$ \\
\hline N1 & $77(15,7)$ & $139(12,5)$ \\
\hline N2 & $148(30,1)$ & $312(28,0)$ \\
\hline N3 & $123(25,1)$ & $359(32,3)$ \\
\hline $\begin{array}{l}\text { N4/ } \\
\text { dring- } \\
\text { lich }\end{array}$ & $135(27,5)$ & $281(25,3)$ \\
\hline $\begin{array}{l}\text { 2Dargestellt sind die Anzahl der Notfalleingriffe } \\
\text { sowie deren prozentualer Anteil }\end{array}$ \\
\hline
\end{tabular}

- Gynäkologie und Geburtshilfe (GYN/GEB),

- Urologie (URO).

Nach Überprüfung auf Normalverteilung mittels Kolmogorov-Smirnov-Test erfolgte die Analyse zur Bestimmung eines statistisch signifikanten Unterschieds zwischen den Gruppen mithilfe des Mann-WhitneyU-Tests unter Angabe von Mittelwert und Standardabweichung ( $\mathrm{MW} \pm \mathrm{SD})$. Für das statistische Signifikanzniveau wurde ein $p$ Wert $\leq 0,05$ festgesetzt. Die statistischen Analysen wurden mit der Statistiksoftware Stata 14.2 durchgeführt.

Die Online-Umfrage wurde im Deutschen Register für Klinische Studien unter der Nummer DRKS00020368 prospektiv registriert; ethische oder berufsrechtliche
Tab. 3 Umsetzungsdauer operativer Notfalleingriffe nach Notfallkategorie gesamt ${ }^{\mathrm{a}}$

\begin{tabular}{|l|l|l|}
\hline- & $\begin{array}{l}\text { Dauer von An- } \\
\text { meldung bis } \\
\text { zum Beginn der } \\
\text { Anästhesie } \\
\text { (in min) }\end{array}$ & $\begin{array}{l}\text { Dauer von An- } \\
\text { meldung bis } \\
\text { zum Schnitt } \\
\text { (in min) }\end{array}$ \\
\hline N0 & $20,7 \pm 14,3$ & $43,6 \pm 31,8$ \\
\hline N1 & $61,5 \pm 48,7$ & $90,1 \pm 56,1$ \\
\hline N2 & $187,9 \pm 152,0$ & $220,5 \pm 153,4$ \\
\hline N3 & $394,5 \pm 392,3$ & $428,3 \pm 397,9$ \\
\hline $\begin{array}{l}\text { N4/ } \\
\text { dring- } \\
\text { lich }\end{array}$ & $494,8 \pm 484,4$ & $519,6 \pm 486,6$ \\
\hline $\begin{array}{l}\text { aDargestellt sind die Mittelwerte in Minuten }( \pm \\
\text { Standardabweichung) }\end{array}$
\end{tabular}

Bedenken seitens der Ethikkommission der Medizinischen Fakultät der Universität Rostock bestanden nicht.

\section{Ergebnisse}

Insgesamt nahmen 26 Krankenhäuser an der Studie teil. Davon wurden 12 Krankenhäuser der Gruppe 1 (5 Häuser der Grundund Regelversorgung sowie 7 Häuser der Schwerpunktversorgung) und 14 Krankenhäuser der Gruppe 2 (Häuser der Maximalversorgung, davon 8 Universitätskrankenhäuser) zugeordnet.

Es wurden insgesamt 1742 Op.-Fälle dokumentiert. Bei 64 Fällen (3,7\%) wurde die Klassifikation im Vergleich zur Erstmeldung nochmals geändert (20-mal Abmeldung, 24-mal Herabstufung und 20-

Hier steht eine Anzeige. 


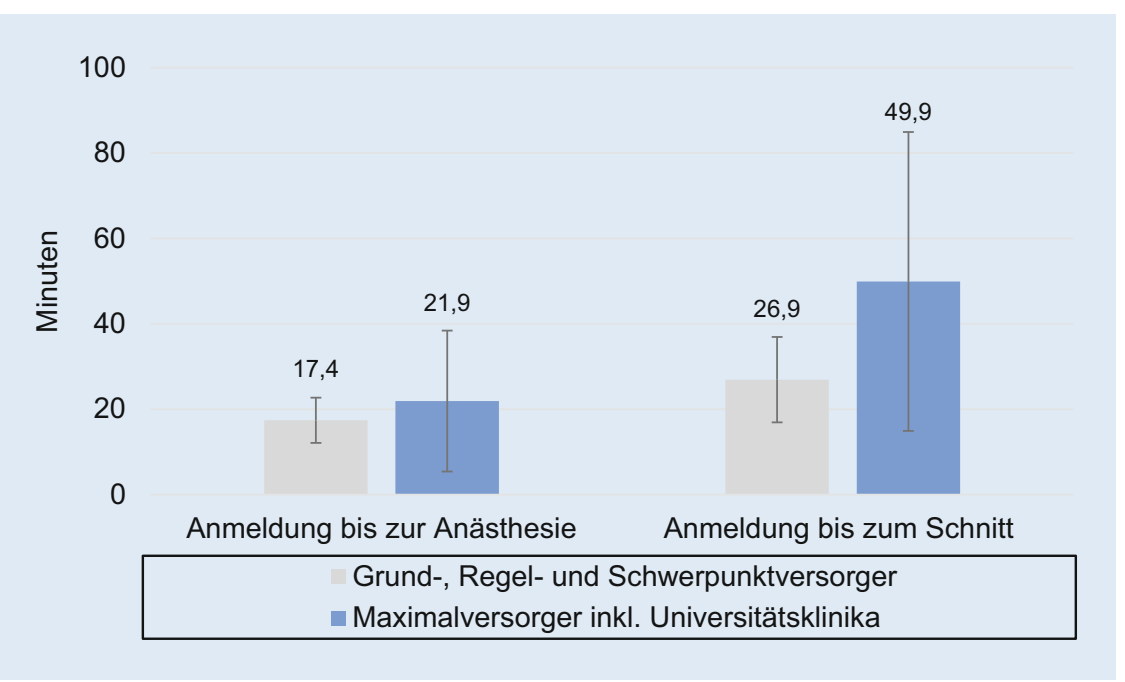

Abb. $3 \Delta$ Vergleich der Umsetzungsdauer von Notfalleingriffen der Kategorie N0 (Eingriff höchster Dringlichkeit). Dargestellt ist die Umsetzungsdauer von der Op.-Anmeldung bis zum Beginn der Anästhesie und bis zum Beginn der Operation (Schnitt)

mal Hochstufung). 75 Datensätze mussten aufgrund von Fehlern in der Dokumentation oder oben genannten Kriterien ausgeschlossen werden: Beginn der Anästhesie bis Schnitt >120 min (22 Fälle), Op.-Anmeldung bis Beginn der Anästhesie außerhalb der in den Ausschlusskriterien definierten Zeitspannen (19 Fälle), Schnitt vor Beginn der Anästhesie (18 Fälle), Beginn der Anästhesie/Schnitt vor Op.Anmeldung (16 Fälle). Der Auswertung liegen insgesamt 1603 Datensätze zugrunde, davon 491 Fälle aus Häusern der Grund-, Regel- und Schwerpunktversorgung sowie 1112 aus Krankenhäusern der Maximalversorgung, einschließlich Universitätskliniken. Die Anzahl der Fälle betrug in den Fachabteilungen Allgemeinchirurgie $n=563$, Unfallchirurgie $n=301$, Gynäkologie und Geburtshilfe $n=214$ sowie Urologie $n=196$. Des Weiteren wurden Op.-Fälle der operativen Disziplinen Neurochirurgie $(n=100)$, Hals-NasenOhren-Heilkunde $(n=65)$, Herzchirurgie $(n=45)$, Kinderchirurgie $(n=41)$, MundKiefer-Gesichtschirurgie $(n=37)$, Orthopädie $(n=28)$ und Augenheilkunde $(n=13)$ eingeschlossen.

Die erhobenen Strukturmerkmale der teilnehmenden Häuser sind in $\mathbf{0}$ Tab. 1 zusammengefasst.

Das operative Geschehen, betrachtet man das Verhältnis von Notfalleingriffe zu Elektiveingriffen, unterschied sich zwischen Maximalversorgern, einschließ- lich Universitätskliniken, $(14,5 \pm 6,3 \%$ vs. $82,6 \pm 8,1 \% ; 2,9 \%$ ohne Zuordnung) nicht signifikant $(p=0,826)$ von dem an Häusern der Grund-, Regel- und Schwerpunktversorgung $(12,3 \pm 6,1 \%$ vs. $86,7 \pm 6,3 \%$; $1,0 \%$ ohne Zuordnung).

Die Anzahl und prozentuale Verteilung der operativen Notfalleingriffe nach Notfallkategorien im Vergleich zwischen Grund-, Regel- und Schwerpunktversorgern einerseits und Maximalversorgern, einschließlich Universitätskliniken, andererseits zeigt $\square$ Tab. 2. Die Anzahl der Fälle NO ist, bedingt durch die spezifische Entität dieser Notfälle, sehr gering. N1 macht ca. $13-15 \%$ der Notfälle aus, die Kategorien N2-N4/dringlich machen jeweils ca. 25-32\% der Notfälle aus. Die Verteilung der Notfallkategorien unterscheidet sich zwischen den beiden untersuchten Gruppen nicht signifikant $(p=0,731)$.

Die Verteilung der Notfallanmeldungen im Tagesverlauf in Abhängigkeit des Versorgungstyps stellt 0 Abb. 1 dar. Sowohl in Krankenhäusern der Grund-, Regel- und Schwerpunktversorgung als auch in Kliniken der Maximalversorgung, einschließlich Universitätsklinika, zeigen sich hierbei Doppelspitzen mit einem hohen Anteil sowohl direkt am Morgen als auch gegen Mittag bis zum frühen Nachmittag. Die Verteilung der einzelnen Notfallklassifikationen zwischen 0 und $24 \mathrm{Uhr}$ zeigt - Abb. 2.
Einen Überblick über die durchschnittliche Dauer, die nach Anmeldung einer Notfall-Op. je nach Klassifikation in deutschen Krankenhäusern bis zum Beginn der Anästhesie bzw. bis zum Schnitt, unabhängig vom Versorgungstyp, vergeht, gibt - Tab. 3.

Für die Notfallklassifikationen N0, N2 und $\mathrm{N} 4 /$ dringlich zeigen sich bei der durchschnittlichen Umsetzungsdauer bis zum Beginn der Anästhesie $(p=0,922$; $p=0,268$ resp. $p=0,408)$ bzw. bis zum Schnitt $(p=0,064 ; p=0,091$ resp. $p=0,187)$ keine statistischen Unterschiede zwischen den Gruppen (• Abb. 3, 4 und 5).

Bei Eingriffen der Klassifikation N1 (sehr hohe Dringlichkeit) ist der Zeitabschnitt zwischen Op.-Meldung und Beginn der Anästhesie in der Gruppe der Maximalversorger, einschließlich der Universitätskliniken, mit einer durchschnittlichen Dauer von 58,9 min signifikant kürzer als in Häusern der Grund-, Regel- und Schwerpunktversorgung, in denen die durchschnittliche Dauer 66,2 min beträgt $(p=0,043)$. Die Auswertung des Zeitraums von der Anmeldung des Eingriffs bis zum Schnitt ergibt für diese Notfallkategorie hingegen keinen signifikanten Unterschied $(p=0,764)$ (• Abb. 6).

In der Kategorie N3 (Durchführung am Ende des Elektivprogramms) ist die durchschnittliche Dauer von der Anmeldung bis zum Beginn der Anästhesie $(p=0,005)$ bzw. bis zum Schnitt $(p=0,003)$ mit 298,6 min resp. 328,0 min in Häusern der Grund-, Regel- und Schwerpunktversorgung signifikant kürzer als in der Gruppe der Maximalversorger, in der durchschnittlich 428,0 min bis zum Anästhesiebeginn sowie $462,7 \mathrm{~min}$ bis zum Beginn der Operation vergehen (ब Abb. 7).

Die detaillierten Ergebnisse der Auswertungen auf Ebene der Fachdisziplinen sind in Tab. 4 zusammengefasst.

In der Allgemeinchirurgie zeigte sich an Häusern der Maximalversorgung, einschließlich Universitätskliniken, für operative Notfälle der Kategorie $\mathrm{N} 1$ zwar ein signifikant kürzeres Umsetzungsintervall von der Op.-Anmeldung bis zum Beginn der Anästhesie (MW 58,1 min vs. 80,1 min; $p=0,037)$, nicht aber beim Schnitt. Allgemeinchirurgische Eingriffe der Klassifikation N3 weisen hingegen in Häusern der Grund-, Regel- und Schwerpunkt- 


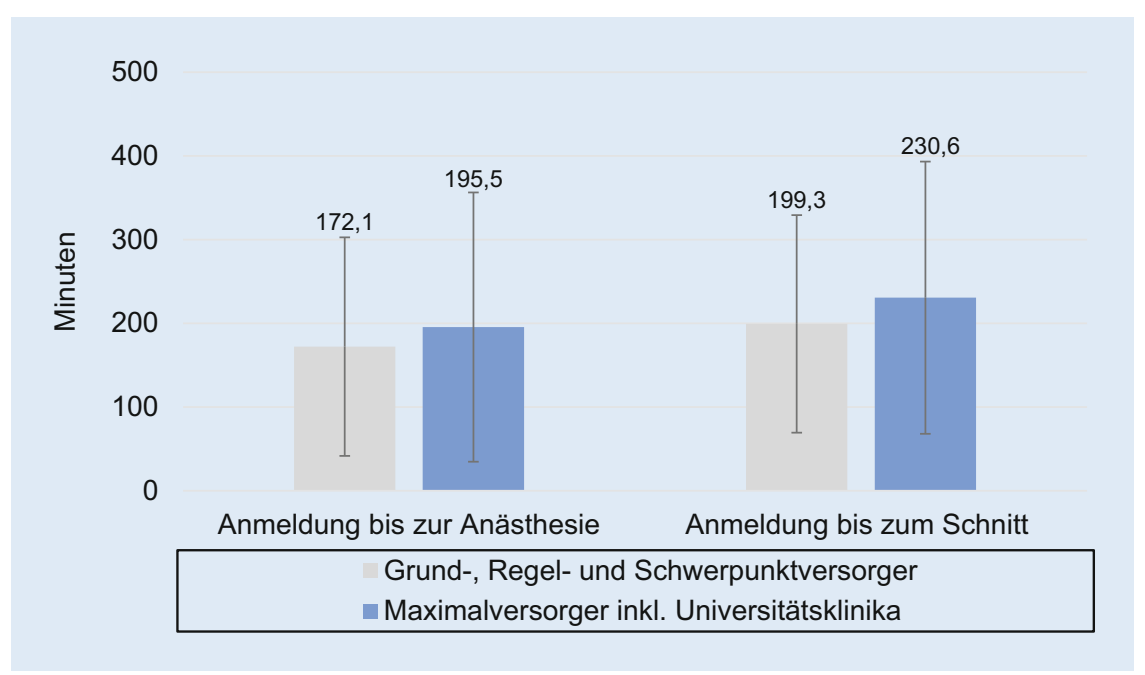

Abb. 4 ム Vergleich der Umsetzungsdauer von Notfalleingriffen der Kategorie N2 (Op.-Beginn $\leq 6 \mathrm{~h}$ nach Meldung). Dargestellt ist die Umsetzungsdauer von der Op.-Anmeldung bis zum Beginn der Anästhesie und bis zum Beginn der Operation (Schnitt)

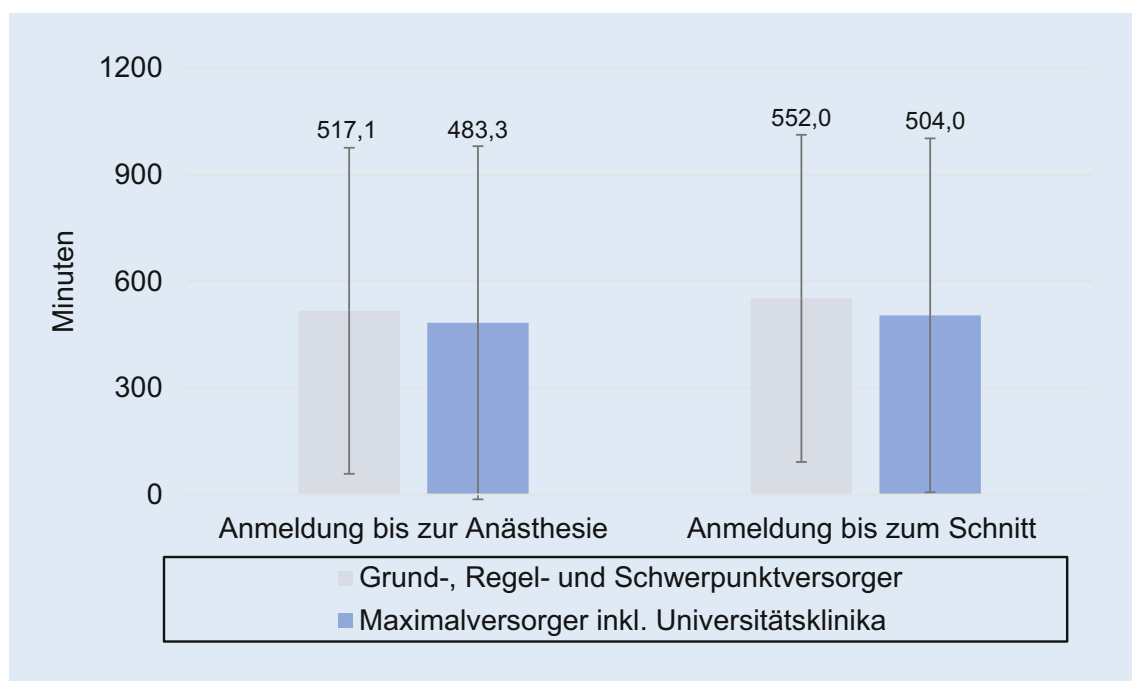

Abb. $5 \Delta$ Vergleich der Umsetzungsdauer von Notfalleingriffen der Kategorie N4/dringlich (Op. innerhalb 12-24h). Dargestellt ist die Umsetzungsdauer von der Op.-Anmeldung bis zum Beginn der Anästhesie und bis zum Beginn der Operation (Schnitt)

versorgung eine signifikant geringere Umsetzungsdauer sowohl beim Anästhesiebeginn (MW 287,8 min vs. $417,1 \mathrm{~min}$; $p=0,045$ ) als auch beim Schnitt (MW $316,3 \mathrm{~min}$ vs. $459,0 \mathrm{~min} ; p=0,032$ ) auf.

Die Umsetzung unfallchirurgischer Eingriffe der Notfallklassifikation N1 erfolgte, bezogen auf den Op.-Schnitt, an Häusern der Grund-, Regel- und Schwerpunktversorgung mit einer durchschnittlichen Dauer von 91,2 min statistisch signifikant schneller als in Häusern der Maximalversorgung mit 133,0 min $(p=0,036)$. von Grund-, Regel- und Schwerpunktversorgern (626,5 min bzw. 645,6 min).

Einen Überblick über die häufigsten Notfalleingriffe je Notfallstufe in Abhängigkeit vom Krankenhaustyp gibt $\bullet$ Tab. 5 .

\section{Diskussion}

Die Empfehlungen von BDA, BDC und VOPM zur Klassifikation von Notfalleingriffen kommen in Krankenhäusern aller Versorgungsstufen in Deutschland zur Anwendung und werden von der großen Mehrheit der OP-Verantwortlichen als hilfreiches Instrument in der OP-Koordination erachtet [9]. Dennoch beschränken sich Informationen zur Häufigkeitsverteilung der verschiedenen Notfallkategorien sowie zu deren zeitlichen Realisierung im klinischen Alltag auf Berichte einzelner Krankenhäuser [8, 13]. Aussagen aus der Untersuchung eines größeren Kollektivs unterschiedlich großer Häuser fehlten hingegen bisher. Die vorliegende Arbeit liefert erstmals eine detaillierte Datenbasis, welche sowohl die Häufigkeit als auch die zeitliche Realisierung operativer Notfalleingriffe in deutschen Krankenhäusern beschreibt.

Frühere Arbeiten haben gezeigt, dass es erhebliche Unterschiede im Prozessmanagement in den OP-Bereichen von Krankenhäusern unterschiedlicher Größe und mit unterschiedlichem akademischen Status in Deutschland gibt. So zeigte sich in größeren Häusern und Universitätskliniken eine signifikant höhere Rate an Fallabsagen von elektiven Op. am Op.-Tag [18] und von Verzögerungen beim morgendlichen Op.-Beginn [12]. Ein weiteres Ziel der vorliegenden Arbeit war somit zu untersuchen, ob es zu relevanten Unterschieden in der Umsetzung des Notfallprogramms in Krankenhäusern unterschiedlicher GröBenordnungen kommt.

Im Ergebnis der Studie kann festgestellt werden, dass das Spektrum ungeplanter Op., gemessen an ihrer Dringlichkeit, trotz Unterschieden in der Anzahl durchzuführender Notfalleingriffe in Deutschland über alle Krankenhaustypen vergleichbar ist. Die Durchführung von Notfall-Op. erscheint dabei im Vergleich zu der zu erwartenden Umsetzungsdauer in ihrer Gesamtheit zeitgerecht, und zwar unabhängig vom Versorgungstyp. In diesen Ergebnis- 


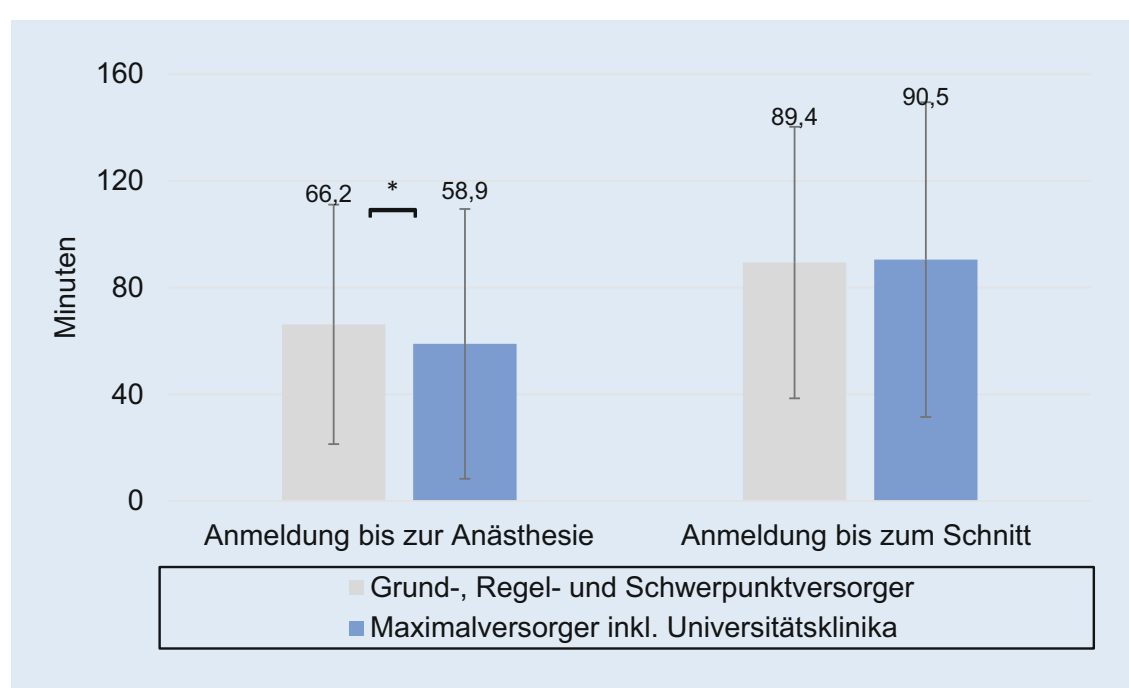

Abb. 6 \ Vergleich der Umsetzungsdauer von Notfalleingriffen der Kategorie N1 (sehr hohe Dringlichkeit). Dargestellt ist die Umsetzungsdauer von der Op.-Anmeldung bis zum Beginn der Anästhesie und bis zum Beginn der Operation (Schnitt). Sternchen $p \leq 0,05$

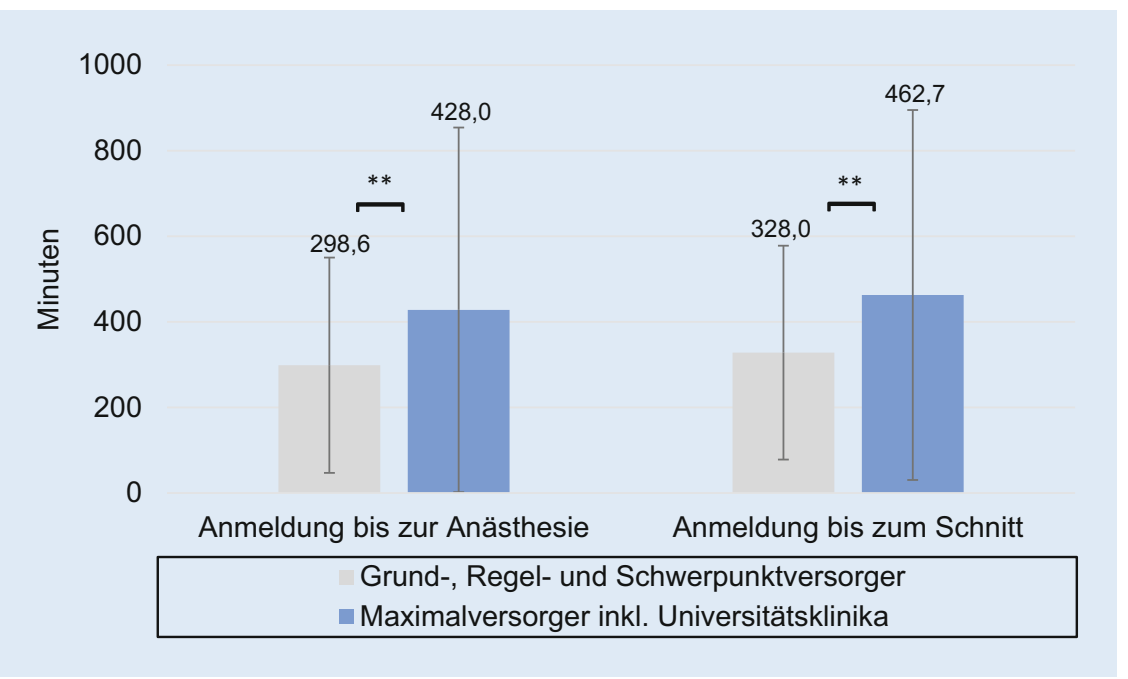

Abb. 7 ^ Vergleich der Umsetzungsdauer von Notfalleingriffen der Kategorie N3 (Op. am Ende des Elektivprogramms). Dargestellt ist die Umsetzungsdauer von der Op.-Anmeldung bis zum Beginn der Anästhesie und bis zum Beginn der Operation (Schnitt). 2 Sternchen $p \leq 0,01$

sen spiegelt sich sowohl die Qualität einer in Deutschland überall gleichwertigen Patientenversorgung als auch die durch Flächendeckung gekennzeichnete deutsche Krankenhausversorgung wider. Der Patient erwartet zu Recht, insbesondere im Notfall, eine zeit- und bedarfsgerechte, nach medizinischen Standards qualitativ hochwertige und qualitätsgesicherte Versorgung [4]. So beträgt die Entfernung ins nächstgelegene Krankenhaus, die ein Patient deutschlandweit im Durchschnitt zurücklegen muss, beispielsweise bei der Notfallindikation zur Implantation einer Endoprothese am Hüftgelenk infolge einer
Fraktur nur $6 \mathrm{~km}$ [14]. Jedoch benötigt es zur Erfüllung dieses Versorgungsauftrages flächendeckend die Vorhaltung enormer Ressourcen, insbesondere für Grund- und Regelversorger. Dieser Spagat führt seit Längerem zu kontroversen Diskussionen, nicht zuletzt vor dem Hintergrund der Untersuchungen der Bertelsmann-Stiftung im Jahr 2019, die einen radikalen Umbau der Krankenhauslandschaft hin zu einer stärkeren Standortkonzentration empfehlen [5].

Welchen Einfluss personelle, strukturelle und organisatorische Ressourcen trotz der insgesamt zeitgerechten Durch- führung auf das Management operativer Notfalleingriffe haben, zeigt sich bei Einzelbetrachtung der Notfallklassifikationen. So spielt bei Operationen der Kategorien N0 und N1 das schnellstmögliche Vorhandensein freier OP-Kapazität, insbesondere im laufenden elektiven Tagesprogramm, eine entscheidende Rolle. Für eine sofortige Umsetzung derartiger Eingriffe sind sowohl zentrale Strukturen mit einer hohen Anzahl an OP als auch personelle Ressourcen zum parallelen anästhesiologischen Beginn der Notfallversorgung, wie diese eher an größeren Krankenhäusern verfügbar sind, von Vorteil. Laut einer Erhebung im Jahr 2015 beträgt die Anzahl vorgehaltener $\mathrm{OP}$ im Durchschnitt in dezentralen OP-Einheiten 3,9 sowie in zentralen OP-Einheiten 6,2, wobei die Varianz mit einem Maximum bis zu $61 \mathrm{OP}$ beträchtlich ist [7]. Dies könnte u.a. den kürzeren Zeitabschnitt von der Anmeldung bis zum Beginn der Anästhesie bei N1-Notfällen in Häusern der Maximalversorgung erklären. Inwieweit die gefundenen Unterschiede für die Behandlungsqualität des einzelnen Patienten klinisch relevant sind, kann auf Basis der Daten der vorliegenden Studie nicht beurteilt werden. In diesem Zusammenhang wird seit Jahren die Notwendigkeit der Vorhaltung eines speziellen Notfall-OP kontrovers diskutiert. Während man bis vor Kurzem davon ausging, dass Kliniken in Deutschland durchschnittlich einen OP explizit zur operativen Versorgung von Notfällen freihalten würden [7], zeigen aktuelle Zahlen aus dem Jahr 2019, dass dies nur noch die Ausnahme (in 3,9\% der Kliniken) zu sein scheint [9]. Unterstützt wird diese Sichtweise von Tschudi et al., nach deren Meinung die Häufigkeit von Notfall-Op. bei der OP-Kapazitätsplanung nicht überbewertet werden sollte [17].

Signifikante Unterschiede zwischen größeren einschließlich universitären Häusern und kleineren Krankenhäusern zeigen sich insbesondere bei Betrachtung von Eingriffen der Klassifikation N3, die entsprechend ihrer Definition bis ans Ende des Elektivprogramms verschoben werden können und folglich den Regelbetrieb nicht beeinflussen. Für die zeitliche Dimension der Umsetzung sind hierbei hauptsächlich organisatorische Belange wie OP-Öffnungszeiten, bestehende 


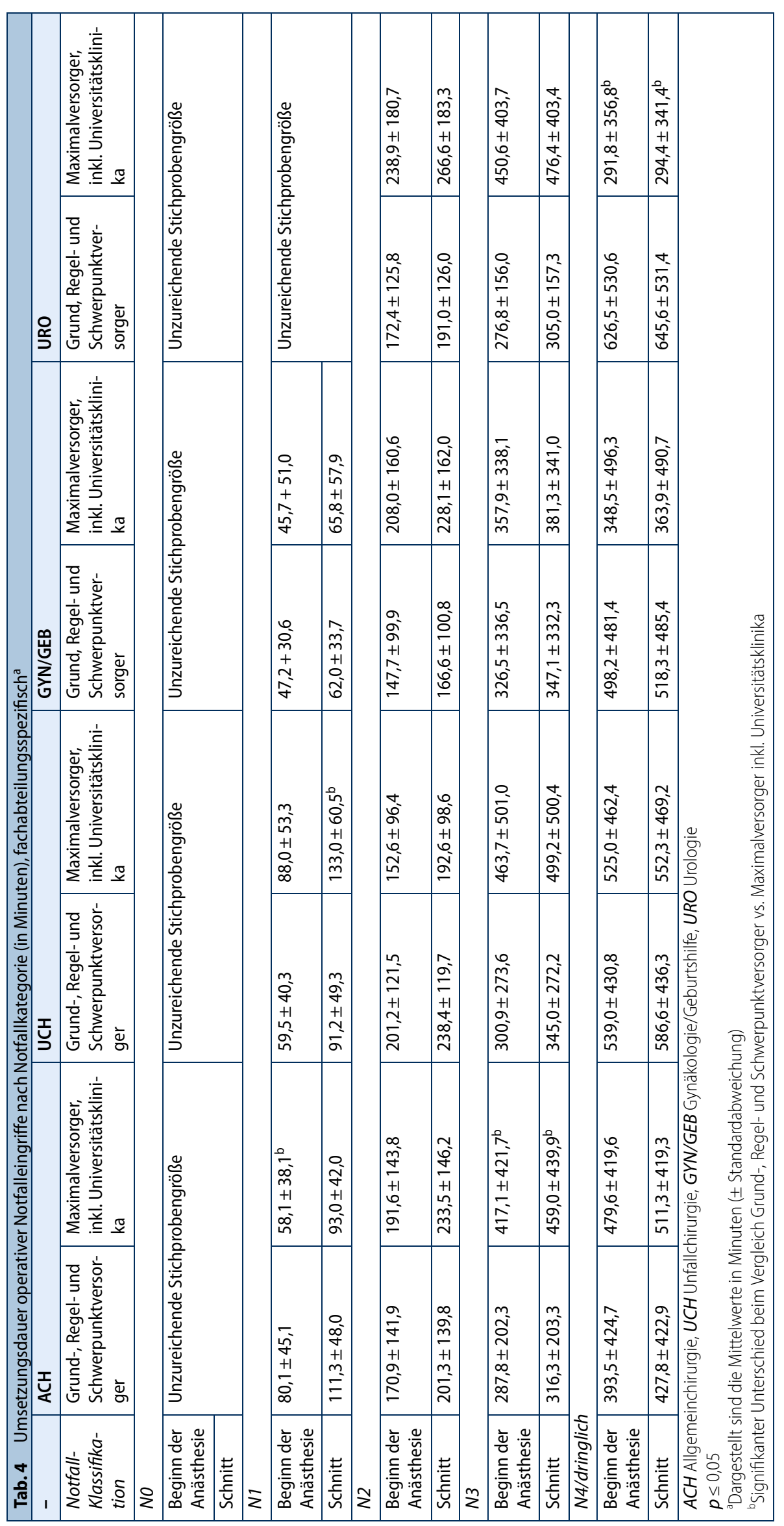


Tab. 5 TOP 3 operativer Notfalleingriffe nach Notfallkategorie ${ }^{a}$

\begin{tabular}{|c|c|c|c|c|}
\hline & \multicolumn{2}{|c|}{ Grund-, Regel- und Schwerpunktversorger } & \multicolumn{2}{|c|}{ Maximalversorger, inkl. Universitätsklinika } \\
\hline No & \multicolumn{2}{|c|}{ Unzureichende Stichprobengröße } & \multicolumn{2}{|c|}{ Unzureichende Stichprobengröße } \\
\hline \multirow[t]{3}{*}{ N1 } & $5-749$ & Sectio caesarea & $5-749$ & Sectio caesarea \\
\hline & $5-541$ & $\begin{array}{l}\text { Laparotomie und Eröffnung des Retroperito- } \\
\text { neums }\end{array}$ & $5-541$ & $\begin{array}{l}\text { Laparotomie und Eröffnung des Retroperito- } \\
\text { neums }\end{array}$ \\
\hline & 8-137 & $\begin{array}{l}\text { Einlegen, Wechsel, Entfernung einer Ureter- } \\
\text { schiene }\end{array}$ & $5-790$ & $\begin{array}{l}\text { Geschlossene Reposition einer Fraktur mit Osteo- } \\
\text { synthese }\end{array}$ \\
\hline \multirow[t]{3}{*}{ N2 } & $5-470$ & Appendektomie & $8-137$ & $\begin{array}{l}\text { Einlegen, Wechsel, Entfernung einer Ureterschie- } \\
\text { ne }\end{array}$ \\
\hline & $8-137$ & $\begin{array}{l}\text { Einlegen, Wechsel, Entfernung einer Ureter- } \\
\text { schiene }\end{array}$ & $5-470$ & Appendektomie \\
\hline & $5-469$ & Op. an Dünn- und Dickdarm & $5-790$ & $\begin{array}{l}\text { Geschlossene Reposition einer Fraktur mit Osteo- } \\
\text { synthese }\end{array}$ \\
\hline \multirow[t]{3}{*}{ N3 } & $5-690$ & Abrasio uteri & $5-790$ & $\begin{array}{l}\text { Geschlossene Reposition einer Fraktur mit Osteo- } \\
\text { synthese }\end{array}$ \\
\hline & $5-470$ & Appendektomie & $5-916$ & Temporäre Weichteildeckung \\
\hline & $5-892$ & Inzision an Haut und Unterhaut & $5-470$ & Appendektomie \\
\hline \multirow{3}{*}{$\begin{array}{l}\text { N4/ } \\
\text { dring- } \\
\text { lich }\end{array}$} & $8-137$ & $\begin{array}{l}\text { Einlegen, Wechsel, Entfernung einer Ureter- } \\
\text { schiene }\end{array}$ & $8-137$ & $\begin{array}{l}\text { Einlegen, Wechsel, Entfernung einer Ureterschie- } \\
\text { ne }\end{array}$ \\
\hline & $5-820$ & $\begin{array}{l}\text { Implantation einer Endoprothese am Hüft- } \\
\text { gelenk }\end{array}$ & $5-399$ & Operationen an Blutgefäßen \\
\hline & $5-794$ & $\begin{array}{l}\text { Offene Reposition einer Mehrfragmentfrak- } \\
\text { tur }\end{array}$ & $5-790$ & $\begin{array}{l}\text { Geschlossene Reposition einer Fraktur mit Osteo- } \\
\text { synthese }\end{array}$ \\
\hline
\end{tabular}

¿Dargestellt sind die OPS-Codes der 3 häufigsten Notfalleingriffe in absteigender Reihenfolge

Konzepte zur Integration von Notfalleingriffen sowie zu Bereitschaftsdienstzeiten ausschlaggebend. Laut Deutschem Krankenhausinstitut (DKI) betreiben 30,5\% der Krankenhäuser wochentäglich ihre OP regelhaft bis zu $8 \mathrm{~h}, 60,9 \%$ zwischen 8 und $10 \mathrm{~h}, 5,3 \%$ bis zu $12 \mathrm{~h}$ sowie $3,2 \%$ länger als $12 \mathrm{~h}$ [7]. Auch wenn bei dieser Erhebung Angaben zum Krankenhaustyp fehlen, ist davon auszugehen, dass gerade die größeren und universitären Häuser über längere OP-Laufzeiten verfügen, was die längere Umsetzungsdauer der N3Meldungen an diesen Häusern erklärt. Diese Annahme wird durch die Angaben der teilnehmenden Häuser unterstützt, nach denen 64,4\% der Maximalversorger/Universitätskliniken, aber nur 33,3\% der kleineren Häuser ihre $\mathrm{OP}$ regulär 10 und mehr Stunden betreiben. Des Weiteren hat der Umgang mit elektiven, infolge von Notfällen verschobenen Programmpunkten Einfluss auf die Durchführung von Notfall-Op. am Ende des Tagesprogramms. Mögliche Vorgehensweisen bestehen in der Berücksichtigung von anteiliger OP-Kapazität für Notfälle bereits in der OP-Planung [2] oder in der Planung eines definierten Zeitintervalls nach Ende der Regel-OP-Laufzeit, in wel- chem verschobene elektive Eingriffe, ggf. interdisziplinär, taggleich noch vor Beginn des Dienstprogramms durchgeführt werden können $[9,11]$.

Auffällig war der geringe Unterschied von Notfällen der Kategorien N3 und N4/ dringlich, insbesondere bei den Maximalversorgern und Universitätskliniken. Der Unterschied zwischen den beiden Kategorien ist, dass bei N3 eine Versorgung am selben Tag (am Ende des Elektivprogrammes) vorgesehen ist, bei N4/dringlich aber auch eine Einplanung in das OP-Programm des Folgetags möglich ist, da die Realisierung erst $24 \mathrm{~h}$ nach Krankenhausaufnahme begonnen sein muss. Dies ist insbesondere für die Versorgung der hüftkopfnahen Frakturen von Bedeutung und dort auch zentraler Bestandteil der Qualitätssicherung. Trotzdem scheinen auch diese N4-Fälle überwiegend noch am selben Tag operiert zu werden, was die hohe Qualität der Notfallversorgung in Deutschland unterstreicht.

Unterschiede bei den N4-Fällen zwischen den Versorgungsstufen zeigten sich insbesondere in der Urologie. Dies mag daran liegen, dass in den Maximal- und Universitätskliniken auch nachts in der Urologie anwesende Ärzte die Op. durchfüh- ren, während in den anderen Häusern aus Gründen der Personalverfügbarkeit die ärztliche Abdeckung über Rufdienste erfolgt und diese Art der nichtdringlichen Notfälle tatsächlich auf den nächsten Tag geschoben werden.

Erfahrungen im klinischen Alltag führen trotz der breiten Akzeptanz der Empfehlungen mitunter zu der Ansicht, dass die definierten Zeitintervalle zur Umsetzung der Notfälle gleichzeitig die Möglichkeit einer subjektiven Auslegung suggerieren könnten [9]. Der Nachweis dieser Annahme, der sich beispielsweise in einer Zunahme von Notfallmeldungen und deren Dringlichkeitsstufen am Ende der regulären OP-Laufzeit zeigen könnte, konnte auf Basis des vorliegenden Datenmaterials nicht erbracht werden.

Die vorliegende Untersuchung ermöglicht erstmals Aussagen zur organisatorischen Versorgungsqualität bei der Umsetzung operativer Notfalleingriffe in deutschen Krankenhäusern. Die Stärke der Untersuchung beruht im Wesentlichen auf ihrem multizentrischen Ansatz, durch welchen erstmals vergleichende Aussagen in Abhängigkeit von der Größe des behandelnden Krankenhauses möglich sind. Aufgrund des begrenzten und im Hinblick 
auf die Verteilung heterogenen Stichprobenumfangs ist die Aussagekraft der Ergebnisse jedoch möglicherweise limitiert und sollte in weiteren Studien verifiziert werden. Kritisch muss bemerkt werden, dass die Erhebung der Studiendaten in einem zwar zufällig gewählten, zeitlich jedoch eng begrenzten Zeitraum durchgeführt wurde. So sind beispielsweise saisonale Effekte bei der Verteilung typischer Notfalldiagnosen, wie sie z. T. in der Unfallchirurgie beschrieben werden [19], nicht auszuschließen. Weitere Untersuchungen zur Thematik des Managements von operativen Notfällen sollten sich perspektivisch mit der im klinischen Alltag gängigen Kategorisierung der für die jeweiligen Fachdisziplinen typischen, häufig auftretenden Notfallindikationen beschäftigen. Die Grundlage für eine derartige Analyse könnten beispielsweise die seit 2009 aus über 320 Krankenhäuser gesammelten Datensätze des Benchmark-Programms der Fachverbände bilden [6].

Fazit für die Praxis

- Die Durchführung von Notfalloperationen (Notfall-Op.) in Deutschland erscheint im Kontext zu den zeitlichen Empfehlungen der Fachverbände insgesamt über alle Versorgungstypen hinweg zeitgerecht.

- Mit Blick auf die Verteilung der einzelnen Notfallklassifikationen unterscheidet sich das operative Notfallaufkommen an Grund-, Regel- und Schwerpunktversorgern nicht wesentlich von dem an Maximalversorgern, einschließlich Universitätskliniken.

- Die Unterschiede im Zeitmanagement bei der Umsetzung von Notfall-Op. sind zwischen Grund-, Regel- und Schwerpunktversorgern im Vergleich zu Maximalversorgern, einschließlich Universitätskliniken, gering.

- Abstufungen auf der Ebene einzelner Notfallkategorien begründen sich durch Unterschiede in den zur Verfügung stehenden Ressourcen wie beispielsweise die Anzahl der OP, einschließlich ihrer Laufzeiten.
Frequency distribution and coordination of emergency operations in German hospitals (FACE). Analysis in 26 hospitals depending on the level of care

Background: Timely emergency surgery is vital as this often has a direct impact on morbidity and mortality. The joint recommendations of the German Associations of Anesthesiologists (BDA), Surgeons (BDC), and Operating Room Management (VOp.M) for coordinative implementation have been available since 2016: N0 (surgery immediately), N1 (surgery start in the next free operating room), N2 (surgery start $\leq 6 \mathrm{~h}$ ), N3 (surgery at the end of the elective schedule), N4/Urgent (surgery within $12-24 \mathrm{~h}$ ). The aim of this study was to describe the situation of care in German hospitals of different sizes for the first time using routine data.

Methods: The data were collected in 26 hospitals with different levels of care over a period of 10 days. The frequency distribution of the individual emergency categories and the duration from the notification of the operation to the start of anesthesia or surgery were examined for the hospital as a whole and for the four operating departments with a typically high ratio of emergencies: general surgery, trauma surgery, gynecology/obstetrics and urology.

Results: A total of 1603 emergency surgical interventions were analyzed. The number of N0 cases was very low due to the specific entity of these emergencies, N1 made up approximately $13-15 \%$ of emergencies and categories N2-N4/Urgent comprised approximately $25-32 \%$ of emergencies each. The average duration between the notification of the operation and the start of anesthesia or surgery was (min): N0 $20.7 \pm 14.3$ and $43.6 \pm 31.8, \mathrm{~N} 161.5 \pm 48.7$ and $90.1 \pm 56.1$, N2 $187.9 \pm 152.0$ and $220.5 \pm 153.4, \mathrm{~N} 3394.5 \pm 392.3$ and $428.3 \pm 397.9$ and N4/Urgent $494.8 \pm 484.4$ and $519.6 \pm 486.6$, respectively. The distribution of the emergency categories did not differ significantly between community hospitals compared to tertiary care hospitals, including university hospitals $(p=0.731)$ and also the duration between notification and start of anesthesia and operation was similar. Significant differences depending on the service level were only found for N1 until the start of anesthesia and for N3 until the start of anesthesia and of surgery. General surgery classified as N3 has a significantly shorter implementation time in community hospitals compared to tertiary care hospitals, including university hospitals, both at the start of anesthesia (mean $287.8 \mathrm{~min}$ versus $417.1 \mathrm{~min} ; p=0.045$ ) and at the start of surgery (mean $316.3 \mathrm{~min}$ versus $459.0 \mathrm{~min} ; p=0.032$ ). The implementation of trauma surgery emergencies classified as $\mathrm{N} 1$ took place, based on the start of surgery in community hospitals with an average duration of $91.2 \mathrm{~min}$, statistically significantly faster than in hospitals with a maximum care of $133.0 \mathrm{~min}(p=0.036)$. In urology, there were notable variations between smaller and larger hospitals in emergency interventions with the classification N4/Urgent for both periods of time, both up to the start of anesthesia $(p=0.012)$ and up to the start of surgery ( $p=0.007)$. At an average of $291.8 \mathrm{~min}$ (start of anesthesia) or $294.4 \mathrm{~min}$ (start of surgery), the implementation time in hospitals with maximum care, including university hospitals, was shorter than in urological clinics of community providers ( $626.5 \mathrm{~min}$ and $645.6 \mathrm{~min}$, respectively). In gynecology/obstetrics, there was no statistically significant difference between the two groups.

Conclusion: Cases with high urgency were surgically treated within a short time period. Overall, differences in time management of emergencies were only small between hospital types. The gradations in the temporal implementation of the individual emergency categories were due particularly to distinctions in the resources available, such as the number of operating theaters, including the run times.

\section{Keywords}

Hospital management - Emergency management - Urgency classification for emergency . Operating room management . Procedural times 


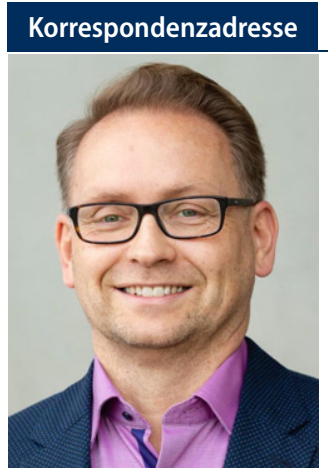

PD Dr. med. Matthias Janda

Klinik und Poliklinik für Anästhesiologie und Intensivtherapie, Universitätsmedizin Rostock Schillingallee 35, 18057 Rostock, Deutschland matthias.janda@med.uni-rostock.de

Funding. Open Access funding enabled and organized by Projekt DEAL.

\section{Einhaltung ethischer Richtlinien}

Interessenkonflikt. M. Janda, O. Karaca, A. Brosin, D. A. Reuter und M. Schuster geben an, dass kein Interessenkonflikt besteht.

Für diesen Beitrag wurden von den Autoren keine Studien an Menschen oder Tieren durchgeführt. Für die aufgeführten Studien gelten die jeweils dort angegebenen ethischen Richtlinien.

Open Access. Dieser Artikel wird unter der Creative Commons Namensnennung 4.0 International Lizenz veröffentlicht, welche die Nutzung, Vervielfältigung, Bearbeitung, Verbreitung und Wiedergabe in jeglichem Medium und Format erlaubt, sofern Sie den/die ursprünglichen Autor(en) und die Quelle ordnungsgemäß nennen, einen Link zur Creative Commons Lizenz beifügen und angeben, ob Änderungen vorgenommen wurden.

Die in diesem Artikel enthaltenen Bilder und sonstiges Drittmaterial unterliegen ebenfalls der genannten Creative Commons Lizenz, sofern sich aus der Abbildungslegende nichts anderes ergibt. Sofern das betreffende Material nicht unter der genannten Creative Commons Lizenz steht und die betreffende Handlung nicht nach gesetzlichen Vorschriften erlaubt ist, ist für die oben aufgeführten Weiterverwendungen des Materials die Einwilligung des jeweiligen Rechteinhabers einzuholen.

Weitere Details zur Lizenz entnehmen Sie bitte der Lizenzinformation auf http://creativecommons.org/ licenses/by/4.0/deed.de.

\section{Literatur}

1. Bauer M, Auhuber TC, Kraus R et al (2020) Glossar perioperativer Prozesszeiten und Kennzahlen. Eine gemeinsame Empfehlung von BDA, BDC,
VOPM, VOPMÖ und SFOPM. Version 2020. Anasth Intensivmed 61:516-531

2. Bauer M, Hinz J, Klockgether-Radke A (2010) Göttinger Leitfaden für OP-Manager. Anaesthesist 59:69-79

3. Bauer M, Waeschle RM, Rüggeberg $J$ et al (2016) Glossar perioperativer Prozesszeiten und Kennzahlen. Eine gemeinsame Empfehlung von BDA / DGAI, BDC / DGCH und VOPM. Version 2016 Anasth Intensivmed 57:669-683

4. Beivers A, Dodt C (2014) Ökonomische Aspekte der ländlichen Notfallversorgung. Notfall Rettungsmed 17:190-198

5. Bertelsmann Stiftung (2019) Zukunftsfähige Krankenhausversorgung. Simulation und Analyse einer Neustrukturierung der Krankenhausversorgung am Beispiel einer Versorgungsregion in NordrheinWestfalen. www.bertelsmann-stiftung.de/de/ publikationen/publikation/did/zukunftsfaehigekrankenhausversorgung.Zugegriffen: 5. Mai 2021

6. Bialas E, Schuster M, Taube C, Diemer M, Bauer M (2014) Fünf Jahre OP-Prozessdaten Benchmarking (2009-2013). Der aktuelle Stand des Programms von VOPM, DGAI/BDA und BDC. Anasth Intensivmed 55:594-613

7. Blum K, Löffert S, Offermanns M, Steffen P (2015) Krankenhausbarometer - Umfrage 2015. Deutsches Krankenhausinstitut, Düsseldorf, S47-48

8. Brosin A, Janda M, Reuter DA, Haas S (2019) Untersuchung zur Häufigkeit und zeitlichen Umsetzung von operativen Notfalleingriffen. Anasth Intensivmed 60(Suppl 12):S499-S522

9. Brosin A, Kropp P, Reuter DA, Janda M (2021) Der Umgang mit Notfallklassifikationen - Wo stehen wir? Ergebnisse einer deutschlandweiten Umfrage. Anaesthesist. https://doi.org/10.1007/ s00101-021-00971-2

10. Destatis (2020) Krankenhaus-Operationen 2019: $38 \%$ der vollstationär behandelten Personen operiert. www.destatis.de/DE/Presse/ Pressemitteilungen/2020/11/PD20_437_231.html. Zugegriffen: 5. Mai 2021

11. Gerst J, Denz C (2021) Notfallintegration. Klassifizierung der OP-Dringlichkeiten (N0-N5). OP Manag Up2date 01:12-17

12. Joos C, Bertheau S, Hauptvogel T et al (2021) Case delay in the OR morning start in hospitals of different size and academic status: results from a German multicenter study to identify incidence and causes of delayed anesthesia ready time. Anaesthesist 70:23-29. https://doi.org/10.1007/ s00101-020-00842-2

13. Karaca $O$ (2018) Notwendige Grundlage für eine valide OP-Planung: Warum eine differenzierte Dokumentation des Notfallaufkommens im OP unverzichtbarist. KU Gesundheitsmanag 87:18-21

14. Klauber J, Geraedts M, Friedrich J, Wasem J (2018) Krankenhaus-Report 2018. Schwerpunkt: Bedarf und Bedarfsgerechtigkeit, 1. Aufl. Schattauer, Stuttgart, $S 81$

15. Kluger Y, Ben-Ishay O, Sartelli M et al (2013) World society of emergency surgery study group initiative on timing of acute care surgery classification (TACS). World J Emerg Surg 8:17

16. Pearse RM, MorenoRP,BauerPetal (2012) Mortality after surgery in Europe: 7 day cohort study. Lancet 380:1059-1065

17. Tschudi $O$, Schüpfer G, Bauer M, Waeschle RM (2017) Effiziente Nutzung von OP-Kapazitäten das Luzerner Konzept. Eine Methodenbeschreibung. Anasth Intensivmed 58:85-93

18. Schuster M, Neumann C, Neumann K et al (2011) The effect of hospital size and surgical service on case cancellation in elective surgery: results from a prospective multicenter study. Anesth Analg 113:578-585

19. von Dercks N, Melz R, Hepp P, Marquass B, Theopold J, Josten C (2011) Saisonale Verteilung von Diagnosen und DRG in der Unfallchirurgie. Unfallchirurg 114:1029-1034 